\title{
NOVEL APPLICATION OF NIH CASE DEFINITIONS IN A PAEDIATRIC TUBERCULOSIS CONTACT INVESTIGATION STUDY
}

Catherine A. Wiseman, ${ }^{1}$ MD, Anna M. Mandalakas, ${ }^{1,2}$ MD, H. Lester Kirchner, ${ }^{3}$ PhD, Robert P. Gie, ${ }^{1}$ MD, H. Simon Schaaf, ${ }^{1} \mathrm{MD}, \mathrm{PhD}$, Elisabetta Walters, ${ }^{1} \mathrm{MD}$, and Anneke C. Hesseling, ${ }^{1} \mathrm{MD}, \mathrm{PhD}$.

Affiliations: ${ }^{1}$ Desmond Tutu TB Centre, Department of Paediatrics and Child Health, Faculty of Medicine and Health Sciences, Stellenbosch University, Cape Town; ${ }^{2}$ Section on Retrovirology and Global Health, Department of Paediatrics, Baylor College of Medicine, Houston Texas and The TB Initiative, Texas Children's Hospital, Houston, Texas, USA; ${ }^{3}$ Division of Medicine, Geisinger Clinic, Danville, PA, USA

Corresponding author: C.A. Wiseman

Desmond Tutu TB Centre

Department of Pediatrics and Child Health

Faculty of Medicine and Health Sciences, Stellenbosch University, PO Box 19063, Tygerberg,

Cape Town, 7505 ,

South Africa

\author{
Alternate corresponding author: A.C Hesseling \\ Desmond Tutu TB Centre \\ Department of Pediatrics and Child Health \\ Faculty of Medicine and Health Sciences, Stellenbosch University, \\ PO Box 19063, Tygerberg, \\ Cape Town, 7505,
}




\section{South Africa}

Word Count: 3062

\section{Abstract: 201 words}

Key Words: Tuberculosis, paediatric, diagnosis, case definition, NIH

Running head title: NIH paediatric TB research definition

Summary: We apply and compare recently proposed International Consensus $(\mathrm{NIH})$ with protocol-specified case definitions for paediatric TB and describe the disease spectrum and severity in a community-based prospective cohort diagnostic TB study using household contact tracing. 
2 Tuberculosis (TB) in children reflects a broad spectrum of disease, ranging from asymptomatic

3 infection through disseminated disease. ${ }^{1}$ Although a higher proportion of young children develop

4 disseminated forms of TB (miliary TB and TB meningitis), ${ }^{2,3}$ primary childhood TB is typically more

5 benign than adult-type TB. The majority ( 75\%) of disease is intrathoracic, ${ }^{4}$ most commonly isolated

6 mediastinal lymph node, Ghon focus or Ghon complex disease. ${ }^{5}$ In mid-childhood, mediastinal lymph

7 node disease predominates; adult-type disease with pleural effusions and cavitation

8 typically emerges during adolescence. ${ }^{4,6}$ The confirmation of TB in children is challenging due

9 patabacillary nature of the disease, challenges in specimen collection and the wide observed

10 disease spectrum. In addition to informing clinical care, the use of standard case definitions is

11 important to enable the adequate comparison of children in clinical research, where the yield of

12 diagnostic tests, response to treatment, and the efficacy of new drugs or regimen and vaccines are

13 evaluated. National Institute of Health (NIH) International Consensus Case Definitions for

14 diagnostic research in childhood TB were proposed in 2012 by an expert panel. ${ }^{7}$ These have not yet

15 bpphed to contact investigations.

16 The yield of diagnostic tests may correlate with disease spectrum and severity. For example, the

17 yield of mycobacterial culture is considerably higher in children with extensive intrathoracic

18 compared to limited disease. ${ }^{8}$ We recently proposed a novel standard approach to describe the

19 spectrum and severity of TB in children ${ }^{5}$ which considers both the extent (containment) of disease

20 and the presence of complications; resulting in a final assignation of "severe" or "nonsevere"

21 disease, applicable to both intrathoracic and extrathoracic TB. ${ }^{5}$

22 The NIH case definition focuses on young children $<10$ years of age presenting with symptoms of

23 intrathoracic TB, typically at the hospital or referral level (Table 1), and was thus expected to have

24 limited applicability to active TB surveillance studies such as household contact tracing. 
25 Prior to the publication of the NIH definition, we developed standard case definitions for use in a

26 community-based household contact tracing study. We applied and compare the NIH and the

27 protocol case definitions and describe the TB disease spectrum and severity ${ }^{5}$ in our prospective

28 community-based study. We hypothesized that our protocol case definition would better apply to

29 our cohort, with anticipated limited disease spectrum and detected through active surveillance.

30

31 


\section{Study design and setting}

34

52

We analysed data from a prospective community-based household contact tracing study aimed at determining the diagnostic utility of 2 commercial Interferon Gamma Release Assays (IGRAs) for detection of Mycobacterium tuberculosis (M.tb) infection and disease in HIV-infected and uninfected children. The study was conducted in three impoverished urban communities with high levels of TB and HIV ${ }^{9}$ in Cape Town, South Africa, consisting of predominantly South African mixed race and Xhosa African populations. The adult TB case notification rate in Cape Town was 671 per 100000 and 315 per 100000 in children aged 0-14 years in 2012 (Personal communication, Judy Caldwell, Cape Town City Health Department).

\section{Eligibility and recruitment}

HIV-infected and -uninfected children aged 3 months to 15 years with and without documented M.tb exposure were recruited between December 2007 and June 2012. Focusing on well children, the study excluded children under $5 \mathrm{~kg}$, with laboratory-documented anemia $(\mathrm{Hb}<9 \mathrm{~g} / \mathrm{dL})$, on antituberculosis therapy, or where consent was not obtained. Infants below $5 \mathrm{~kg}$ were excluded due to blood volumes. Children on isoniazid preventive therapy (IPT) were not excluded. Enrolment was deferred if a Mantoux tuberculin skin test (TST) had been placed within 12 weeks, live attenuated vaccination had been given within 6 weeks, or if there was an acute severe respiratory, diarrheal or neurologic illness.

53 A three-pronged approach was employed, recruiting children via either community TB clinics as household contacts of adult TB cases (who started treatment within preceding 3 months; i.e from 
55 "TB households"), or from neighbouring households ("community controls") or from community 56 paediatric HIV clinics (HIV-infected children with and without documented M.tb exposure).

58 All symptomatic adult household members were screened for TB and if diagnosed with TB, that household was reclassified. Children were investigated at enrolment and follow-up for M.tb 60 infection and disease, using standard protocols. ${ }^{10,11}$

\section{Study measures}

63 Using methods previously described, ${ }^{12}$ the larger study enrolled 1093 children with median follow64 up duration of 15 months. ${ }^{13}$ Children were seen at baseline (enrolment), months $3,6,15$, and in the 65 case of HIV-infected children, 27 months' follow-up. From the total enrolled, a dataset was compiled 66 of all potential TB cases, detected at any (scheduled and unscheduled) study visits.

67 Children were included in analysis of potential TB cases if they were documented as having 68 Confirmed, Probable or Possible TB (protocol definition), reported by the study team, parent or 69 healthcare provider (TB clinic or referral hospital), or if there was laboratory evidence of M.tb, at any 70 time during the study period. Data sources included the study database, clinic, hospital, and routine 71 laboratory surveillance data.

72 Standard methods were used to define TB symptoms, ${ }^{11}$ M.tb contact history, chest radiograph 73 interpretation ${ }^{14}$ and bacteriology. TB disease spectrum and severity were described; ${ }^{5}$ the most 74 severe manifestation was reported. HIV testing was completed on all children with unknown or negative status (Abbott Determine HIV-

$761 / 2$ rapid test followed by DNA PCR or HIV ELISA depending on whether they were younger or older

77 than 18 months, respectively, in the case of a positive or indeterminate test). All HIV-infected

78 children had access to combination anti-retroviral therapy (CART). 
79 TB exposure history was captured using information including the presence of contact with a known

80 adult (> 18 years old) household or other TB source case (person currently receiving TB treatment

81 regardless of sputum smear status or disease type), the level of exposure and the number of TB

82 cases in the household. The level of exposure was reported as a contact score of 1-10 derived from

83 combined data assuming that the level of exposure was a product of proximity, duration and

84 infectivity of exposure. ${ }^{12}$

Measures of TB infection included the TST and IGRAs. The TST (2 TU PPD RT 23, Statens Serum

Institute) was administered intradermally and read using the ballpoint method and callipers at 48-72

hours. TST was completed at all study visits except at month 6 , in the case of a previous reported

TST adverse event, or TST administered within the preceding 3 months. A positive TST was defined as an induration of $\geq 10 \mathrm{~mm}$ in HIV-uninfected and $\geq 5 \mathrm{~mm}$ in HIV-infected children. The T-SPOT.TB completed at all study visits.. ${ }^{17}$ IGRA results were reported at the visit closest to the TB episode as positive (if either QTF or T-SPOT.TB was positive), negative (both tests negative or only 1 available result, which was negative), indeterminate or not done. M.tb infection was defined as either a positive TST and/or IGRA.

TB symptoms and signs were classified at the time of the TB episode (Table 1). $7,11,13,18,19$

Bacteriological data included specimen type, number of specimens sent, number of positive specimens, result of direct smear microscopy for acid-fast-bacilli (AFB), liquid mycobacterial culture (mycobacterial growth indicator tubes; MGIT, Becton Dickinson, Sparks, MD, USA), ${ }^{20-28}$ histology, speciation and drug susceptibility test (DST) pattern using the Genotype ${ }^{\circledR}$ MTBDRplus line probe assay (Hain Lifescience, Nehren, Germany). ${ }^{29}$ Gastric aspirates were collected in all children younger than 5 years ${ }^{13}$ and expectorated sputum samples in all older children at baseline and at subsequent 
105 scheduled or unscheduled study visits where clinically indicated (e.g. new TB symptoms, new

106 reported TB contact, or previous reported abnormality). ${ }^{13}$ Samples were collected, transported and

107 processed following standard guidelines and standard cross-contamination prevention measures

108 were taken. ${ }^{30}$ PCR identification of M.tb on any culture-positive specimen defined bacteriological

109 confirmation, while caseating necrosis/granulomas with or without positive microscopy for AFB on

110 cytology or histology specimens defined histological confirmation.

111 Radiological data included all available chest radiograph (CXR) reports. Antero-posterior and lateral

112 CXR were done at all initial visits and at subsequent study visits as clinically indicated. CXRs were

113 reviewed by two experienced independent blinded reviewers using a standard classification. ${ }^{14}$

114 Where discrepancies arose, a third blinded, independent reviewer was used. Reviewers reported

115 CXRs as being "Certain TB," "Uncertain TB", "Not TB" or "Normal”. A final radiological classification

116 was assigned as either "Compatible with TB," "Abnormal but not compatible with TB" or "Normal".

117 In the case of an abnormal CXR deemed not to be compatible with TB, an alternative clinical

118 diagnosis, where possible, was assigned.

\section{TB disease definitions}

121 The NIH consensus definition ${ }^{7}$ and protocol case definition were used (Table 1). The NIH case definition classified disease as Confirmed, Probable, Possible, Unlikely or Not TB. ${ }^{7}$ Protocol-specified case definitions assigned these same case definitions as a function of bacteriological confirmation, well-defined symptoms/signs, TB-compatible CXR, and TB exposure (Table 1). Tests of infection were purposefully excluded since they were the index test under evaluation in the study.

127 TB episodes were classified as "prevalent" (detected within 6 weeks of enrolment) or "incident" (detected 128 after 6 weeks' enrolment). 
129 All protocols were approved by the Health Research Ethics Committee, Stellenbosch University, and 130 the institutional review boards of Case Western Reserve University, USA, Baylor College of Medicine,

131 USA and the Charite Institute, Berlin, Germany and local health authorities. All well children with

132 M.tb contact or positive TST who were HIV-infected or below 5 years of age were referred for IPT, as

133 per local and international guidelines. ${ }^{31,32}$ All cases of suspected TB were referred for treatment.

\section{Data management and analysis}

135 Agreement between diagnostic approaches was measured using the Kappa statistic with 95\% confidence 136 intervals (CI). All cases with a potential TB diagnosis in our cohort were included in analysis, regardless of 137 age. Odds ratios (OR), calculated with Chi-squared or Fisher's exact tests, were used to compare disease 138 severity in HIV-infected vs. uninfected children and in different age and TB exposure groups. The TB disease 139 spectrum was described. TB incidence rates were calculated by age, HIV and TB exposure status. Data was 140 analysed using STATA/SE version 12.0 (StataCorp LP, Texas, USA). 
Of 1093 children enrolled, 169 were HIV-infected and 924 were HIV-uninfected; 671 (61\%) were enrolled from TB households, 242 (22\%) from neighbouring households, 180 (16\%) children from community HIV clinics, of whom 163 were HIV-infected and 17 were uninfected siblings. Of the remaining 6 HIV-infected children, 5 were recruited from TB households and 1 from a neighbouring household.

There were 111 potential TB disease episodes documented in 109 children, of whom 23 (21\%) were HIV-infected. 62 (56\%) were prevalent and 49 (44\%) incident cases. There was known TB exposure in 82 (74\%) episodes. The median M.tb contact score at enrolment in the 109 children was 5 (0-10). Of prevalent cases eligible for IPT according to programme criteria, only 5/40 (12.5\%) children were on IPT at the time of TB diagnosis; whilst 5 of 18 (28\%) of incident cases had received IPT at diagnosis. A further 6 (33\%) were referred but either failed to attend the clinic or defaulted therapy. 16/22 (72\%) children with a potential TB episode had documented TB exposure but were not referred for IPT based on programme criteria (HIV-uninfected and older than 5 years of age). Of HIV-infected children, $83 \%$ were on CART at the time of TB diagnosis.

Based on NIH case definitions, ${ }^{7}$ there were 8 episodes of confirmed (Table 3), 12 of probable, 17 of possible, 3 of unlikely and 2 of not TB disease. Applying protocol-defined case definitions, there were 23 episodes of confirmed (Table 3), 36 of probable, 27 of possible, 0 of unlikely and 21 of not TB. Of the 111 potential TB disease episodes, 69 were unclassifiable ( 4 due to insufficient data and 65 due to lack of symptoms), of whom 18 (26\%) had no documented TB exposure, using the NIH definition. Four TB episodes were unclassifiable using the protocol case definition due to insufficient data; 2 (50\%) who had no documented TB exposure. Agreement between the NIH and protocol-defined approach was $0.30(95 \% \mathrm{Cl}: 0.23 ; 0.38)$. 
167 Using the protocol case definition and excluding the episodes classified as "not TB," and "unlikely TB," there 168 were 62 episodes (72\%) of nonsevere and 24 episodes (28\%) of severe disease. Of those with severe disease, 169 all had intrathoracic disease, 2 (6\%) had cavities/adult-type disease, 4 (13\%) had expansile pneumonia; and 1 170 (4\%) had disseminated disease (miliary TB) [Table 4]. HIV-infected children were more likely to have severe disease than HIV-uninfected children (OR: 3.87; 95\% $\mathrm{Cl} 1.26 ; 11.81, \mathrm{p}=0.0056$ ) Children with a documented

172 TB source case were less likely to have severe disease than those without known exposure (21\% vs. 44\%; OR:

$1730.34,95 \% \mathrm{Cl}: 0.12 ; 1.01, \mathrm{p}=0.025)$. When stratifying by TB exposure and HIV status, proportions with severe 174 disease remained unchanged, making it challenging to elucidate whether HIV infection or the lack of documented TB exposure determined TB disease severity. The proportion of children with severe disease was similar in children below 2 years of age vs. older children ( $27 \%$ vs. $22 \%$; OR: $0.93,95 \% \mathrm{Cl}: 0.29 ; 2.71$, $\mathrm{p}=0.88) .9$ of the 24 children with severe disease were eligible for IPT based on programme criteria; only 3 of these had received IPT. Children with known TB exposure at enrolment were more likely to have prevalent TB (OR: $1.48,95 \% \mathrm{Cl}: 0.55 ; 3.96, \mathrm{p}=0.38)$ than those without exposure.

180

TB incidence rates (per 1000 patient years) were 109/1000 and 76/1000 in HIV-infected versus uninfected children; $106 / 1000$ and 76/1000 in children under versus over two years; $111 / 1000$ and 53/1000 in children under versus over five years old, and 86/1000 and 74/1000 in those with versus without known M.tb exposure. 
186 This is the first published application of the NIH diagnostic definition for intrathoracic childhood

187 TB, published in $2012 .^{7}$ In our community-based diagnostic study, we found that almost two-thirds

188 offildren could not be classified using this definition, despite the presence of severe disease in almost

189 a third. In contrast, almost all children could be classified using the protocol case definition, which

190 did not rely on the presence of presenting symptoms.

191 This is most likely explained by the emphasis in the NIH approach on diagnosing TB in symptomatic children, not typically seen in contact investigation studies, where the majority of diseased children are expected to have limited disease and thus more limited symptomatology. The NIH approach would thus have limited applicability to paediatric TB studies using active surveillance, e.g. TB contact investigation, and vaccine trials. ${ }^{33}$ We propose the use of our protocol definition as more relevant to contact investigation studies.

There was, understandably, poor agreement between the case definitions. Despite the fact that the protocol case definition did not consider tests of TB infection, almost all children were classifiable using well quantified M.tb exposure as a proxy for tests of infection. Well quantified exposure was previously shown to correlate well with tests of infection and suggested as a possible replacement for tests of infection in resource-poor settings to guide targeted IPT delivery. ${ }^{12}$ A major difference resulting from the use of the $\mathrm{NIH}$ and protocol case definitions lies in the number of confirmed cases detected (Table 3). Using the protocol definition, there were almost threefold the number of confirmed cases than with the NIH definition. Only 8 of these 23 cases (with at least bacteriological confirmation) had "well-defined symptoms"18, although most had symptoms of more acute duration with or without abnormal CXR. We agree that all 23 children may not have had overt disease, however, all were treated as TB by the program. Recent well-documented M.tb exposure with isolated bacteriological confirmation may be described as "acute TB infection" as supported by natural history studies, where positive mycobacterial cultures were obtained in children with 
210 normal chest radiographs and a positive TST or history of M.tb exposure. ${ }^{4}$ We appreciate that whilst

211 the clinical relevance in children with bacteriological confirmation without well-defined TB

212 symptoms remains unknown and an area of ongoing discussion, it is not imprudent to regard such

213 cases with high suspicion due to the risk of dissemination in very young or HIV-infected children,

214 given that laboratory contamination can be excluded. Management should be on a case-by-case

215 basis until consensus is reached.

The site of TB disease seen in our cohort was almost exclusively intrathoracic, but with a wide observed disease spectrum and severity, ranging from uncomplicated intrathoracic lymph node disease to uncontrolled intrathoracic disease with complications (lymph node or other) [Table 4]. Despite this being a predominantly (62\%) asymptomatic (i.e. no "well-defined" symptoms ${ }^{18}$ ) cohort of children recruited through active surveillance, $28 \%$ of children had severe intrathoracic disease. HIV-infected children had the highest proportion of severe disease (48\%), followed by children without documented TB source exposure (44\%). Possible explanations for the unexpectedly high rate of severe disease are the high HIV prevalence and the high proportion of young children in our study (Table 2), both risk factors for severe disease. ${ }^{2,3,34,35} \mathrm{HIV}$ infection is associated with a higher incidence of severe intrathoracic disease including cavities ${ }^{34}$ although not more disseminated disease. ${ }^{34}$ HIV-infected children recently started on CART may however be prone to more severe disease manifestations including TB Immune Reconstitution Inflammatory Syndrome (IRIS). ${ }^{36}$ High TB incidence rates have been reported in HIV-infected children ${ }^{37,38}$ despite the availability of IPT and improved immunological function on $\mathrm{CART}^{39}$ consistent with our findings.

Diagnostic and treatment delay in adults ${ }^{40}$ may also have led to delayed presentation of children resulting in disease progression, especially in children without known TB exposure, where TB may not have been readily suspected in the absence of a known source case. ${ }^{41}$ Lastly, poor IPT uptake may be reflected in the high TB rates seen here in HIV-infected and -uninfected under-five year olds, despite IPT recommendations. Although IPT would not have prevented the majority of our TB cases, 
235 which were diagnosed within 6 weeks of enrolment, IPT at the time of source case identification by

236 the program could have prevented a substantial proportion of disease in child contacts. Active

237 contact tracing and routine IPT delivery in these communities was documented as limited with no

238 structured IPT adherence support offered by the TB program. ${ }^{42,43}$ We have subsequently supported

239 the implementation of structured IPT delivery tools to improve uptake and adherence. ${ }^{44}$

240 


\section{CONCLUSIONS}

242 The current NIH case definition for paediatric intrathoracic TB has limited applicability to household

243 contact studies where, as shown in this study from a high-TB burden setting, a surprisingly wide

244 spectrum of TB disease was observed in HIV-infected and uninfected children. Further work is

245 needed to develop paediatric TB case definitions to ensure that the wide spectrum of relevant

246 paediatric TB observed in clinical research is captured, including in contact investigation studies. 


\section{ACKNOWLEDGEMENTS}

The authors declare that they have no conflicts of interest. This work was supported by the National Institute of Allergy and Infectious Disease at the National Institutes of Health (R01A076199).

We would like to thank the Desmond Tutu TB Centre Paediatric TB team, as well as the study communities, participants, their parents and the local health authorities for their contribution to this study. Funding for this study was received from the NIH, the German Research Foundation (DFG), the Norwegian Programme for Development, Research and Education (NUFU) and the Thrasher Research Foundation. 


\section{REFERENCES}

1. Marais BJ, Gie RP, Schaaf HS, Hesseling AC, Enarson DA, Beyers N. The spectrum of disease in children treated for tuberculosis in a highly endemic area. Int J Tuberc Lung Dis 2006;10:732-

8.

2. Marais BJ, Donald PR, Gie RP, Schaaf HS, Beyers N. Diversity of disease in childhood pulmonary tuberculosis. Ann Trop Paediatr 2005;25:79-86.

3. Marais BJ, Gie RP, Schaaf HS, et al. The natural history of childhood intra-thoracic tuberculosis: a critical review of literature from the pre-chemotherapy era. Int J Tuberc Lung Dis 2004;8:392-402.

4. Donald PR, Ahmed A, Burman WJ, et al. Requirements for the clinical evaluation of new antituberculosis agents in children. The international journal of tuberculosis and lung disease : the official journal of the International Union against Tuberculosis and Lung Disease 2013;17:794-9.

5. Wiseman CA, Gie RP, Starke JR, et al. A Proposed Comprehensive Classification of Tuberculosis Disease Severity in Children. Pediatr Infect Dis J 2012;31:347-52.

6. Weber HC, Beyers N, Gie RP, Schaaf HS, Fish T, Donald PR. The clinical and radiological features of tuberculosis in adolescents. Annals of tropical paediatrics 2000;20:5-10.

7. Graham SM, et al Evaluation of Tuberculosis Diagnostics in Children: 1. Proposed Clinical Case Definitions for Classification of Intrathoracic Tuberculosis Disease. Consensus From an Expert Panel. Journal of Infectious Diseases 2012;2012:S199-S208.

8. Marais BJ, Hesseling AC, Gie RP, Schaaf HS, Enarson DA, Beyers N. The bacteriologic yield in children with intrathoracic tuberculosis. Clin Infect Dis 2006;42:e69-71.

9. Kritzinger FE, den Boon S, Verver S, et al. No decrease in annual risk of tuberculosis infection in endemic area in Cape Town, South Africa. Trop Med Int Health 2009;14:136-42. 
10. Marais BJ, Gie RP, Hesseling AC, Schaaf HS, Enarson DA, Beyers N. Radiographic signs and symptoms in children treated for tuberculosis: possible implications for symptom-based screening in resource-limited settings. Pediatr Infect Dis J 2006;25:237-40.

11. Marais BJ, Gie RP, Hesseling AC, et al. A refined symptom-based approach to diagnose pulmonary tuberculosis in children. Pediatrics 2006;118:e1350-9.

12. Mandalakas A, Kirchner HL, Lombard C, Walzyl G, Gie Rp, Hesseling AC. Well Quantified TB Exposure is a Reliable Surrogate Measure of M.tb Infection in children. IJTLD 2012;16 1033-

9.

13. Mandalakas AM, Hesseling AC. Study Title: The Utility of Interferon-Gamma Release Assays in TB-HIV co-infected Children. 2011.

14. Marais BJ, Gie RP, Schaaf HS, et al. A proposed radiological classification of childhood intrathoracic tuberculosis. Pediatr Radiol 2004;34:886-94.

15. Lalvani A, Pathan AA, McShane H, et al. Rapid detection of Mycobacterium tuberculosis infection by enumeration of antigen-specific T cells. Am J Respir Crit Care Med 2001;163:824-8.

16. Connell TG, Rangaka MX, Curtis N, Wilkinson RJ. QuantiFERON-TB Gold: state of the art for the diagnosis of tuberculosis infection? Expert Rev Mol Diagn 2006;6:663-77.

17. Mandalakas AM, van Wyk S, Kirchner HL, et al. Detecting tuberculosis infection in HIVinfected children: a study of diagnostic accuracy, confounding and interaction. The Pediatric infectious disease journal 2013;32:e111-8.

18. Marais BJ, Gie RP, Obihara CC, Hesseling AC, Schaaf HS, Beyers N. Well defined symptoms are of value in the diagnosis of childhood pulmonary tuberculosis. Arch Dis Child 2005;90:1162-5.

19. Cuevas LE, et al. Evaluation of Tuberculosis Diagnostics in Children: 2. Methodological Issues for Conducting and Reporting Research Evaluations of Tuberculosis Diagnostics for 
Intrathoracic Tuberculosis in Children. Consensus From an Expert Panel. Journal of Infectious Diseases 2012;205:S209-S15.

20. Lee JJ, Suo J, Lin CB, Wang JD, Lin TY, Tsai YC. Comparative evaluation of the BACTEC MGIT 960 system with solid medium for isolation of mycobacteria. The international journal of tuberculosis and lung disease : the official journal of the International Union against Tuberculosis and Lung Disease 2003;7:569-74.

21. Zaruba R, Kralova M. [Evaluation of the effectiveness of the BACTEC MGIT automatic system for culture of mycobacteria in comparison with classical methods of culture. Experience after one year of use]. Epidemiol Mikrobiol Imunol 2002;51:66-70.

22. Ardito F, Sanguinetti M, Sechi L, et al. Comparison of the mycobacteria growth indicator tube with radiometric and solid culture for isolation of mycobacteria from clinical specimens and susceptibility testing of Mycobacterium tuberculosis. New Microbiol 2000;23:151-8.

23. Badak FZ, Kiska DL, Setterquist S, Hartley C, O'Connell MA, Hopfer RL. Comparison of mycobacteria growth indicator tube with BACTEC 460 for detection and recovery of mycobacteria from clinical specimens. Journal of clinical microbiology 1996;34:2236-9.

24. Flanagan PG, Williams R, Paull A. Comparison of two automated systems for the isolation of mycobacteria from clinical specimens. Eur J Clin Microbiol Infect Dis 1999;18:912-4.

25. Hanna BA, Ebrahimzadeh A, Elliott LB, et al. Multicenter evaluation of the BACTEC MGIT 960 system for recovery of mycobacteria. Journal of clinical microbiology 1999;37:748-52.

26. Tortoli E, Cichero P, Piersimoni C, Simonetti MT, Gesu G, Nista D. Use of BACTEC MGIT 960 for recovery of mycobacteria from clinical specimens: multicenter study. Journal of clinical microbiology 1999;37:3578-82.

27. Tsuyuguchi K, Ikeda T, Nakatani K, Tsuboi T, Sato A, Kurasawa T. [Evaluation of the Mycobacteria Growth Indicator Tube system for detection and quantification of mycobacteria from clinical specimens]. Kekkaku 2003;78:389-93. 
28. Whyte T, Cormican M, Hanahoe B, Doran G, Collins T, Corbett-Feeney G. Comparison of BACTEC MGIT 960 and BACTEC 460 for culture of Mycobacteria. Diagn Microbiol Infect Dis 2000;38:123-6.

29. Hain Lifescience Genotype MTBDRplus homepage. Available at: http://www.hainlifescience.de/en/products/microbiology/mycobacteria/genotype-mtbdrplus.html Accessed 7 March 2013.

30. Carroll NM, Richardson M, van Helden PD. Criteria for identification of cross-contamination of cultures of Mycobacterium tuberculosis in routine microbiology laboratories. J Clin Microbiol 2003;41:2269; author reply 269-70.

31. Republic of South Africa Department of Health. South African National Tuberculosis Management Guidelines, 2009. . Available at http://familymedicineukznacza/Libraries/Guidelines Protocols/TB Guidelines 2009sflbashx Accessed 23 June 20132009.

32. World Health Organisation. Guidelines for treatment of tuberculosis, fourth edition. Available at http://wwwwhoint/tb/publications/2010/9789241547833/en/indexhtml Accessed 23 June 20132010.

33. Hatherill M, Verver S, Mahomed H. Consensus statement on diagnostic end points for infant tuberculosis vaccine trials. Clinical infectious diseases : an official publication of the Infectious Diseases Society of America 2012;54:493-501.

34. Schaaf HS, Marais BJ, Whitelaw A, et al. Culture-confirmed childhood tuberculosis in Cape Town, South Africa: a review of 596 cases. BMC Infect Dis 2007;7:140.

35. Madhi SA, Huebner RE, Doedens L, Aduc T, Wesley D, Cooper PA. HIV-1 co-infection in children hospitalised with tuberculosis in South Africa. Int J Tuberc Lung Dis 2000;4:448-54.

36. Bakeera-Kitaka S, Conesa-Botella A, Dhabangi A, et al. Tuberculosis in human immunodeficiency virus infected Ugandan children starting on antiretroviral therapy. The 
international journal of tuberculosis and lung disease : the official journal of the International Union against Tuberculosis and Lung Disease 2011;15:1082-6.

37. Hesseling AC, Cotton MF, Jennings $\mathrm{T}$, et al. High incidence of tuberculosis among HIVinfected infants: evidence from a South African population-based study highlights the need for improved tuberculosis control strategies. Clin Infect Dis 2009;48:108-14.

38. Walters E, Cotton MF, Rabie H, Schaaf HS, Walters LO, Marais BJ. Clinical presentation and outcome of tuberculosis in human immunodeficiency virus infected children on antiretroviral therapy. BMC Pediatr 2008;8:1.

39. Madhi SA, Nachman S, Violari A, et al. Primary isoniazid prophylaxis against tuberculosis in HIV-exposed children. The New England journal of medicine 2011;365:21-31.

40. Van Wyk SS, Enarson DA, Beyers N, Lombard C, Hesseling AC. Consulting private health care providers aggravates treatment delay in urban South African tuberculosis patients. The international journal of tuberculosis and lung disease : the official journal of the International Union against Tuberculosis and Lung Disease 2011;15:1069-76.

41. Golub JE, Bur S, Cronin WA, et al. Delayed tuberculosis diagnosis and tuberculosis transmission. The international journal of tuberculosis and lung disease : the official journal of the International Union against Tuberculosis and Lung Disease 2006;10:24-30.

42. Van Wyk SS, Hamade H, Hesseling AC, Beyers N, Enarson DA, Mandalakas AM. Recording isoniazid preventive therapy delivery to children: operational challenges. Int J Tuberc Lung Dis 2010;14:650-3.

43. van Wyk SS, Reid AJ, Mandalakas AM, et al. Operational challenges in managing Isoniazid Preventive Therapy in child contacts: a high-burden setting perspective. BMC public health 2011;11:544.

44. Van Soelen N, Van Wyk S, Du Preez K, et al. Operational Implementation of an IPT register in a high-burden setting. As presented at Symposium 42 at the 43rd Union World Conference on Lung Health; Kuala Lumpur, Malaysia, November 2012. IJTLD 2012;16:S64. 
Table 1. Definition and comparison between NIH and protocol case definitions for pediatric tuberculosis

\begin{tabular}{|c|c|c|c|}
\hline $\begin{array}{l}\text { Disease } \\
\text { categories }\end{array}$ & NIH Case definition ${ }^{7}$ & Protocol Case definition & Key differences \\
\hline Confirmed TB & $\begin{array}{l}\geq 1 \text { symptom/sign }{ }^{7} \text { together } \\
\text { with microbiological } \\
\text { confirmation of } \\
\text { Mycobacterium tuberculosis } \\
\text { (M.tb) }\end{array}$ & $\begin{array}{l}\text { Bacteriological or histological }{ }^{1} \\
\text { confirmation of M.tb, either } \\
\text { 1.alone, }{ }^{2} \text { (with/without TB } \\
\text { exposure/ alternative (non-TB) } \\
\text { CXR diagnosis), or with } \\
\text { 2. either a TB-compatible CXR, } \\
\text { or } \\
\text { 3. well-defined } \\
\text { symptoms/signs }{ }^{3,4}\end{array}$ & $\begin{array}{l}\text { Bacteriological } \\
\text { confirmation alone } \\
\text { considered and symptoms } \\
\text { not mandatory in protocol } \\
\text { case definition. } \\
\text { Additional symptoms and } \\
\text { signs considered in the } \\
\text { protocol case definition. }\end{array}$ \\
\hline Probable TB & $\begin{array}{l}\geq 1 \text { symptom/sign and a TB- } \\
\text { compatible CXR and: } \\
\text { 1.either a positive } \\
\text { treatment response, or } \\
\text { 2.documented exposure to } \\
\text { M.tb, or } \\
\text { 3. immunological evidence } \\
\text { of M.tb infection }\end{array}$ & $\begin{array}{l}\text { 1. Nonspecific histology }{ }^{5} \text { with } \\
\text { 1.1. a TB-compatible CXR, or } \\
\text { 1.2. symptoms/signs, or } \\
\text { 2. A TB-compatible CXR with } \\
\text { 2.1. symptoms/signs, or } \\
\text { 2.2. TB exposure }\end{array}$ & $\begin{array}{l}\text { Symptoms/signs not } \\
\text { mandatory in protocol } \\
\text { definition; tests of } \\
\text { infection and treatment } \\
\text { response not considered in } \\
\text { protocol case definition. } \\
\text { Additional symptoms and } \\
\text { signs considered in the } \\
\text { protocol case definition. }\end{array}$ \\
\hline Possible TB & $\begin{array}{l}\geq 1 \text { symptom/sign and either } \\
\text { 1.a positive treatment } \\
\text { response or documented } \\
\text { exposure to M.tb or } \\
\text { immunological evidence of }\end{array}$ & $\begin{array}{l}\text { Either of the following: } \\
\text { 1. nonspecific histology, or } \\
\text { 2. a TB-compatible CXR, or } \\
\text { 3. symptoms/signs } \\
\text { with or without TB exposure }\end{array}$ & $\begin{array}{l}\text { Symptoms/signs not } \\
\text { mandatory in protocol } \\
\text { definition; TB-compatible } \\
\text { CXR alone is defined as } \\
\text { possible TB in protocol }\end{array}$ \\
\hline
\end{tabular}




\begin{tabular}{|c|c|c|c|}
\hline & $\begin{array}{l}\text { M.tb infection, or: } \\
\text { 2. A TB-compatible CXR }\end{array}$ & & $\begin{array}{l}\text { case definition; tests of } \\
\text { infection and treatment } \\
\text { response not considered in } \\
\text { protocol case definition. } \\
\text { Additional symptoms and } \\
\text { signs considered in the } \\
\text { protocol case definition. }\end{array}$ \\
\hline Unlikely TB & $\begin{array}{l}\geq 1 \text { symptom/sign but not } \\
\text { fitting any of above } \\
\text { definitions, but with no } \\
\text { alternative established } \\
\text { diagnosis }\end{array}$ & $\begin{array}{l}\text { Abnormal, TB-incompatible CXR } \\
\text { with no alternative radiological } \\
\text { or clinical diagnosis, with or } \\
\text { without TB exposure }\end{array}$ & $\begin{array}{l}\text { Symptoms/signs not } \\
\text { mandatory in protocol } \\
\text { case definition, otherwise } \\
\text { similar. } \\
\text { Additional symptoms and } \\
\text { signs considered in the } \\
\text { protocol case definition. }\end{array}$ \\
\hline Not TB & $\begin{array}{l}\geq 1 \text { symptom/sign, not fitting } \\
\text { any of the above } \\
\text { definitions, and with an } \\
\text { established alternative } \\
\text { diagnosis }\end{array}$ & $\begin{array}{l}\text { Abnormal,TB-incompatible CXR } \\
\text { with an alternative radiological } \\
\text { or clinical diagnosis, with or } \\
\text { without TB exposure }\end{array}$ & $\begin{array}{l}\text { Symptoms/signs not } \\
\text { mandatory in protocol } \\
\text { definition, otherwise } \\
\text { similar }\end{array}$ \\
\hline
\end{tabular}

${ }^{1}$ Histological confirmation implies Ziel Nielsen Stain (Zn) positive with granulomatous inflammation and/or caseating necrosis

${ }^{2}$ Area of ongoing clinical debate/discussion

${ }^{3}$ Symptoms/signs include any or more than one of the following

Cough: persistent, non-remitting cough for more than 2 weeks, not responding to a course of antibiotics

Weight loss/failure to thrive: unexplained weight loss of $>5 \%$ vs. the highest recorded weight /clear deviation from previous growth trajectory \pm documented crossing of centile lines in preceding 3 months \pm weight for age $z$-score of $\leq-2$ with no previous/recent growth trajectory information, not responding to nutritional rehabilitation/cART 
Persistent unexplained fever: subjectively reported by guardian and a temperature of $>38$ degrees Celsius for more than one week objectively recorded at least once.

Persistent, unexplained reduced playfulness or activity: perceived and reported by the parent or caregiver.

${ }^{4}$ Protocol defined symptoms and signs are any of the above as well as or any of the following, in isolation:

Neck nodes/visible neck swelling: defined as noticed by the parent/caregiver in the preceding month

Night sweats: reported by caregiver of a drenching nature, requiring a change of clothing.

Convulsions, lethargy or a decreased level of consciousness: defined as reported by the caregiver in the preceding two

weeks

${ }^{5} \mathrm{Zn}$ - with granulomatous inflammation and/or caseating necrosis 
Table 2. Characteristics of children enrolled in a community-based diagnostic study ( $N=1093$ children) at enrolment and at the time of diagnosis of a potential disease episode ( $N=109)$

\begin{tabular}{|c|c|c|}
\hline Characteristics & Total cohort $(\mathrm{N}=1093)$ & Children with potential TB ( $\mathrm{N}=109)$ \\
\hline $\begin{array}{l}\text { Age at enrolment (months: } \\
\text { median, range) }\end{array}$ & $61(3-190)$ & $39(5-184)$ \\
\hline Age at TB episode & N/A & $41(5-188)$ \\
\hline Under 2 years of age [N (\%)] & $197(18)$ & $26(23)$ \\
\hline Under 5 years age $[\mathrm{N}(\%)]$ & $534(49)$ & $74(67)$ \\
\hline HIV-infected [N (\%)] & $169(15)$ & $23(21)$ \\
\hline $\begin{array}{l}\text { Median CD4 count at enrolment: } \\
\text { Absolute (range), \% (range) }\end{array}$ & 1400 (35-4200), $28.5(3-47)$ & 1450 (35-3700), 27 (3-44) \\
\hline $\begin{array}{l}\text { Median CD4 count at TB episode: } \\
\text { Absolute (range), \% (range) }\end{array}$ & N/A & 1232 (35-2601), 27 (3-47) \\
\hline $\begin{array}{l}\text { Follow-up - HIV-infected children } \\
\text { (months: median, range) }\end{array}$ & $15(0-29)$ & $15(3-28)$ \\
\hline $\begin{array}{l}\text { Follow-up HIV-uninfected children } \\
\text { (months: median, range) }\end{array}$ & $15(0-21)$ & $15(0-21)$ \\
\hline Ethnicity: Mixed race [N (\%)] & $717(66)$ & $73(67)$ \\
\hline Ethnicity: Xhosa [N (\%)] & $365(33)$ & $35(32)$ \\
\hline Ethnicity: Other [N (\%)] & $11(1)$ & $1(1)$ \\
\hline Community A [N (\%)] & $355(32)$ & $28(26)$ \\
\hline Community B [N (\%)] & $359(33)$ & $45(41)$ \\
\hline Community C [N (\%)] & $379(35)$ & $36(33)$ \\
\hline Recruited from TB household [N & $671(61)$ & $76(70)$ \\
\hline
\end{tabular}




\begin{tabular}{|l|l|l|}
\hline$(\%)]$ & & \\
\hline Recruited from neighbouring & $242(22)$ & $13(12)$ \\
household [N (\%)] & & \\
\hline $\begin{array}{l}\text { Recruited from community HIV } \\
\text { clinic [N (\%)] }\end{array}$ & $180(16)$ & $20(18)$ \\
\hline
\end{tabular}


Table 3. Comparison of diagnostic features in children with confirmed tuberculosis as classified by the NIH and protocol case definitions

\begin{tabular}{ll}
\hline Confirmed TB based on the NIH definition $(\mathrm{N}=8)$ & $\mathrm{N}(\%)$
\end{tabular}

Bacteriological confirmation and symptoms

Bacteriological confirmation, symptoms and a lobar pneumonia on CXR

Bacteriological confirmation, symptoms and documented TB exposure

Bacteriological confirmation, symptoms, TB exposure together with interstitial

pneumonia on CXR

Bacteriological confirmation, symptoms, TB exposure and a TB-compatible CXR

Confirmed TB based on the protocol definition ( $\mathrm{N}=\mathbf{2 3}$ )

Bacteriological confirmation alone

Bacteriological confirmation (MDR M.tb) with multiple palpable cervical lymph

nodes (1.5 cm diameter)

Bacteriological confirmation with interstitial pneumonia on CXR

Bacteriological confirmation with, lobar pneumonia and acute respiratory

symptoms

Bacteriological confirmation and TB exposure ("acute infection")

Bacteriological confirmation, TB exposure and an interstitial pneumonia on CXR

Bacteriological confirmation, TB exposure, lobar pneumonia on CXR and acute

respiratory symptoms

Bacteriological confirmation, TB exposure and a TB-compatible CXR

Bacteriological confirmation and well-defined symptoms

Bacteriological confirmation, well-defined symptoms and interstitial pneumonia on CXR 
exposure

Bacteriological confirmation, well-defined symptoms, documented TB exposure

and interstitial pneumonia on CXR

Bacteriological confirmation, well-defined symptoms, documented TB exposure

1 (4)

and a TB-compatible CXR 
Table 4. Tuberculosis disease spectrum observed in children with confirmed, probable and possible tuberculosis based on the protocol case definition ( $N=86$ )

$\mathrm{N}(\%$ of $\mathrm{N}=86)$

Intrathoracic tuberculosis

-Isolated uncomplicated intrathoracic lymph node disease

-Controlled, uncomplicated intrathoracic (parenchymal) disease $(n=29)$ and 3 with

additional uncomplicated intrathoracic lymph node disease

-Pleural effusion with uncomplicated intrathoracic lymph node disease

-Complicated intrathoracic lymph node disease ( 8 in isolation); 2 had additional

controlled uncomplicated intrathoracic (parenchymal) disease

-Uncontrolled uncomplicated intrathoracic (parenchymal) disease, of which 2 had

additional uncomplicated intrathoracic lymph node disease

-Disseminated disease (miliary TB) with bilateral small pleural effusions and

uncomplicated intrathoracic lymph node disease

-Uncontrolled complicated intrathoracic disease (complications were mostly

lymph node related)

Extrathoracic tuberculosis

-Controlled uncomplicated cervical adenitis

Note: Entities listed show where there was overlap between more than one disease entity. Disease extent (controlled vs. uncontrolled) and the presence of complications are described using a standard approach used to describe disease spectrum and severity. ${ }^{11}$ 